\title{
New Power Mechanism Design in ZDY1900S Type All Hydraulic Tunnel Drilling Rig
}

\author{
Yanbiao $\mathrm{Li}^{1, \mathrm{a}}$,Shicheng Zheng ${ }^{2, \mathrm{~b}}$,Jie Zhang ${ }^{3, \mathrm{c}}$,Zhe Zhang ${ }^{4, \mathrm{~d}}$, \\ LaCang, ${ }^{5, \mathrm{e}}$ ShucanHe $\mathrm{e}^{6, \mathrm{f}}$ \\ ${ }^{1}$ China University of Mining \&Technology(Beijing)CUMTB Beijing 100083 china \\ ${ }^{\mathrm{a} C u m t b 947 @ 126 . c o m,}$ \\ ${ }^{b}$ shichengzhengs163.com, ${ }^{c} 472134409 @ q q . c o m,{ }^{d} 1373649745 @ q q . c o m,{ }^{e} 1872185448 @ q q . c o m,{ }^{f} 5$ \\ 74019819@qq.com
}

Keywords: drilling rig; exploration; tunnel; all hydraulic; new power mechanism

\begin{abstract}
Tunnel exploration is a kind of method with high efficiency and economy, but special tunnel exploration drilling rigs are scarce. ZDY1900S type all hydraulic tunnel drilling rig is a kind of equipment designed for tunnel exploration, also can be used to drill gas drainage holes, water exploration holes and other engineering holes. The design approach, mechanical system and hydraulic system for the drilling rig were introduced. Improvement was carried out based on it, design idea of double-power institution was introduced and work principle was described in detail.
\end{abstract}

\section{Introduction}

With the rapid development of coal science and technology in recent years, coal mining technology and mining equipment also got strong development. Due to the complexity of the coal mine construction conditions, sticking and buried drilling accidents are prone to happen, which makes the drilling tool buried in a hole with huge economic losses. In order to solve this problem, this paper designed the hydraulic tunnel drilling rig power head matching for ZDY1900S type coal mine to handle work. Power head mechanism designed in this paper is improved based on ZDY1900S drill to determine the main parameters of the drilling rig.

\section{Selection of Main Parameters}

Drill Pipe Diameter.Due to the largerdrillingdiameter, the direction of drill must be accurate. Therefore, thedrill pipe diameter has a lot to do with thedriling diameter gradation, the control effect of directional drilling and the intensity of labor. Determining the two kinds of drill pipe with the diameter of $77 \mathrm{~mm}$ and $89 \mathrm{~mm}$, we choose drill pipe by replacing slips.

Main Shaft Output Speed.When drilling into the near-horizontal directional drilling hole, drill pipe and the wall of hole have seriousfrictions.Drill power consumption is also large. Although high speed can improve the times of drill head cutting rock inunit time and increase the drilling efficiency, it does more harm than goodcompared with the increase of tool wear and power consumption. According to the solid parameters of ZDY1900S typedrill, the maximum speed is $220 \mathrm{r} / \mathrm{min}$ and the minimum speed is decided by the minimum stable flow of hydraulic system ${ }^{[1]}$.

Feeding and Pull outAbility. With thepoorcoal mine condition, especially in the construction of near-horizontal hole, hole collapse and chipping are prone to happen in the hole,so drill should have a strong ability to deal with the accident. Therefore, drill must have bigger pulling forcethan normal circumstances.The biggest force of the original drill is $77 \mathrm{kN}$.Combiningthe structure design of feed device, the largest feeding and pulling force is $150 \mathrm{kN}$, which can handle general sticking and buried drilling accidents.

\section{Innovation of the New Power Head}

Background of the New Power Head Theory. In the coal mine gas drilling, it is recommended to use water as flushing medium. When drilling, it uses mud pump to inject water into drill hole as a 
borehole circulation medium. In soft coal seam, due to the erosion and washing of high pressure water, outside hole wall of the drill is vulnerable to collapse and fall, which is unable to form a good hole wall.

Technical Requirements of the New Power Head. Compared with major gyrator, this newly equipped gyrator needs lower rotate speed and smaller torque, because it just needs to ensure that drill pipe could rotate endlessly instead of providing drilling power. As the core component of this new power head, this auxiliary gyrator chooses to use variable displacement motor with slow rotate speed and large torque to obtain an adjustment scope with a ratio from 1 to 3.47 rather than installing the mechanical gear shift mechanism ${ }^{[2,3]}$.

Fig.1.Gearbox layout

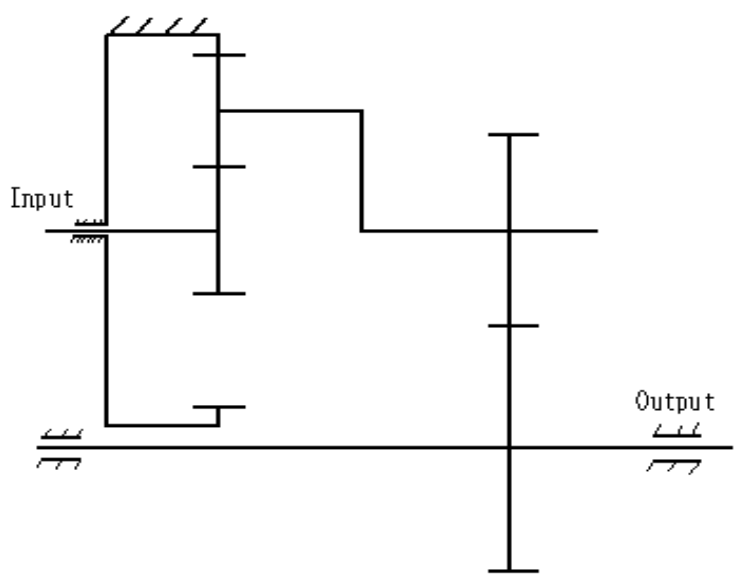

Gear shift mechanism: it should meet the needs of output rotate speed and ensure that the drill pipe could successfully spin. There should be a certain speed difference between the major gyrator and the auxiliary gyrator, thus the auxiliary gyrator needs larger transmission ratio, choosing a transmission case with gear reduction ratio about 20. See Fig.2.It chooses plan three-axis frame and put NGW type planetary gear reducer as the first-level transmission, of which the compact structure shortens the distance between center of gyration and guide surface and improves the force condition when layer board moves ${ }^{[4,5]}$.

Hydraulic chuck: it adopts rubber sleeve chuck instead of force-amplifier with high transmission efficiency; it has compact structure and large tightening force; it has small external dimension and rotational inertia; the chuck has a long service life and large clamping range, thus when the drill pipe is worn, its clamping force will not be effected.

Advantage of the New Power Head. This new power head is provided oil by various hydraulic pumps, and consequently it could work independently and avoid unnecessary oil circuit interference, making the rig work in a more stable condition. The design of the new power head ensures that the drill pipe works endlessly and effectively avoid collapse and blocking in the hole and jamming of the drilling tool, improving work efficiency and reducing unnecessary troubles. 


\section{Rig Working Process of the New Power Head}

Based on the current pipe changing mode, the author designs a pipe changing process mainly depended on the interaction of power heads. The working process is as follows ${ }^{[6]}$

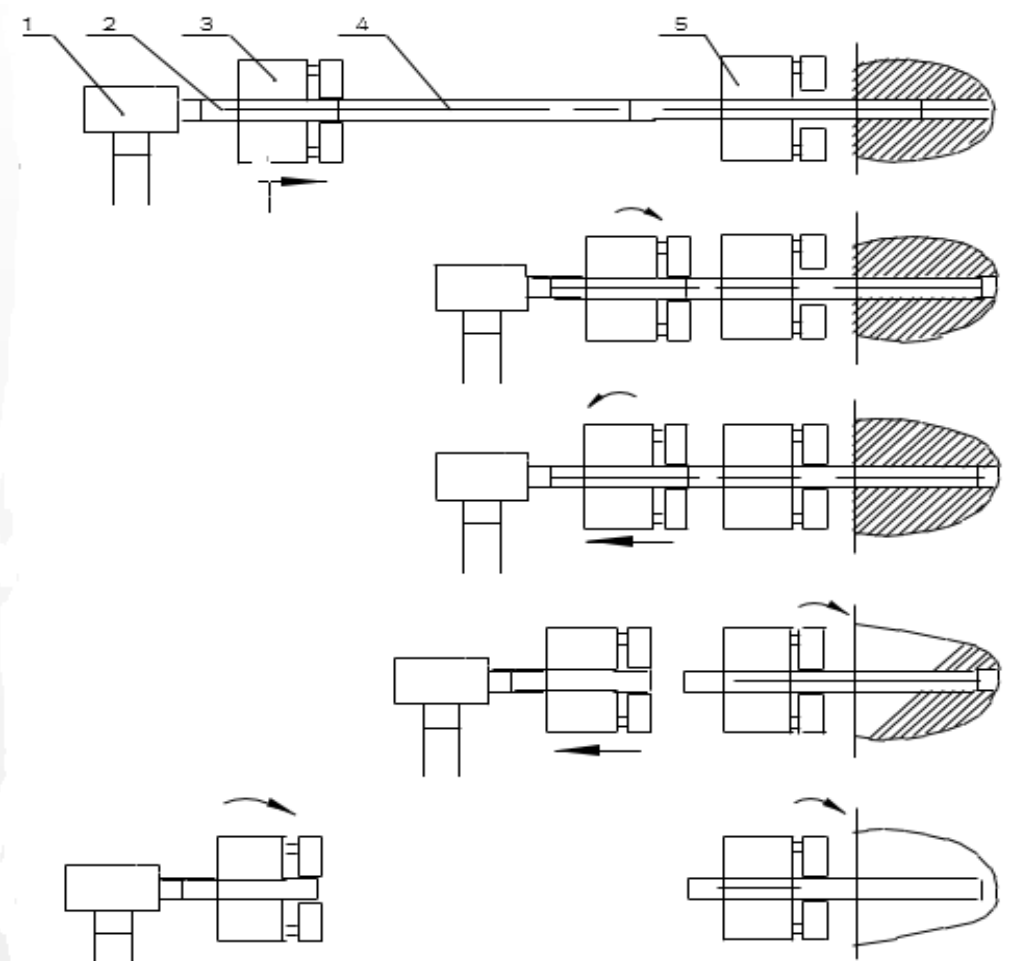

1.water tap 2.specific small drill pipe 3.major power head 4.drill pipe 5.new power head 6.coal bed

Fig. 2 The flow diagram of automatic changing pipe

1. The rig is in normal drilling process when it starts. The major power head clamps the specific drill pipe and rotate and feed constantly. The new power head is now in loosening condition.

2. When the rig drilling into the maximum stroke, the holder of the new power head begins to clamp the drilling pipe in the coal bed.

3. The major power head stops rotating and pulls out constantly and then backs up to the end of the process. While the new power head still rotates in a low speed, the drill pipe in the coal bed rotating endlessly.

4. When the major power head reaches to the initial end of the process (there is a travel switch), the automatic pipe changing mechanism begins to work. It takes out one drill pipe from drill pipe warehouse, and then put the drill pipe in the central axis between the two power heads. The new power head still works in a low speed now.

5. Turn into another rotate cycle.

\section{Conclusion}

This paper introduces the whole mechanism and each part of the mechanism. Due to the deficiency in current rig pipe changing, the author comes up with the theory of double power head mechanism, and describes the whole working process in detail. The rig adopts double power head, thus it can achieve pipe changing without stopping working and reduce accidents such as jamming of the drilling tool and tools burying due to stopping working when changing pipe. The double power head adopts demountable design with basic components combined flexibly, meeting working requirements in a better way. 


\section{References}

[1] Ying Xinsheng, Tian Hongliang:MK-7All Hydraulic Tunnel Drilling Rig(Exploration Engineering 2003)

[2] Yao ke,Ying Xinsheng: Design and Application of ZDY4000SAll Hydraulic Tunnel Drilling Rig (Coal Engineering 2006)

[3] S. Strom, M.K.Balov, H. Kjorholt, R. Gasso, E.H. Vefring, and R. Rommetweit. Future drilling technology: closer than you think. World Oil, October 2008.

[4] Sun Jigang: Mechanical Design. (Higher Education Press,Beijing 2005)

[5] Sun,Cheng Zuomo: Mechanical Principles. (Higher Education Press, Beijing 2006)

[6] Wang Bin: Design and Sesearch of Automatic Changing Pipe Mechanism of New Head Drill Machine (China University of Mining and Technology, China 2014) 